\title{
Aprovechamiento del escapo y los botones florales de AGAVE kerchovel en el Valle de Tehuacán-Cuicatlán, México
}

\author{
Paulina Brena-Bustamante" ${ }^{1,3}$, Rafael Lira-Saade², Edmundo García-Moya ${ }^{1}$, \\ Angélica Romero-Manzanares ${ }^{1}$, Héctor Cervantes-Maya ${ }^{2}$, Martín López-Carrera ${ }^{2}$ \\ y Salvador Chávez-Herrera ${ }^{2}$ \\ ${ }^{1}$ Colegio de Postgraduados, Montecillo, Estado de México, México \\ ${ }^{2}$ Facultad de Estudios Superiores Iztacala, Universidad Nacional Autónoma de México, \\ Tlalnepantla, Estado de México, México \\ ${ }^{3}$ Autor para la correspondencia: paubrebu@hotmail.com
}

\begin{abstract}
Resumen: La Reserva de la Biósfera del Valle de Tehuacán-Cuicatlán es una provincia de gran riqueza etnoflorística en la que resalta el género Agave por su diversidad específica y los múltiples recursos que ofrece a los lugareños. Agave kerchovei Lem. es una especie de interés particular por el aprovechamiento de la estructura floral. El objetivo de este estudio fue obtener información acerca del conocimiento y uso tradicional de los recursos derivados de A. kerchovei en San Rafael, Puebla y San Gabriel Casa Blanca, Oaxaca, México. Se realizaron entrevistas a hombres y mujeres relacionados con las actividades del hogar y del campo, a quienes se les aplicó un cuestionario estructurado con tres apartados: (1) identificación por medio de fotografías de la especie y las estructuras aprovechadas, (2) lugar, forma de recolecta y uso y (3) cantidad y frecuencia de recolecta de A. kerchovei. Este agave es conocido como cacayas y rabo de león. Las estructuras que se utilizan son los botones florales (capullos), como alimento tanto para humanos como para el ganado ovicaprino; la forma de uso es muy variada y su recolecta es particular. Los entrevistados indicaron que sus sitios de acopio son cercanos a sus asentamientos y las recolectas se realizan durante los meses de octubre a diciembre; en los cuales se cosechan hasta siete quiotes, acudiendo en más de cinco ocasiones. La información sobre A. kerchovei es escasa, de tal forma que este estudio amplía su conocimiento tradicional e importancia cultural.
\end{abstract}

Palabras clave: agaves, cacayas, etnobotánica, rabo de león.

\begin{abstract}
The Tehuacán-Cuicatlán Biosphere Reserve is a province of great richness etno-floristic which highlights the genus Agave for its specific diversity and multiple resources offered to residents. Agave kerchovei Lem. is a species of particular interest in the use of floral structure. The aim of this study was to obtain information about the traditional use and knowledge of the resources derived from A. kerchovei in San Rafael, Puebla, and San Gabriel Casa Blanca, Oaxaca, Mexico. Group interviews were conducted with men and women related to home and fields activities, who were applied a structured questionnaire with three sections: (1) identification by photographs of the species and utilized structures, (2) place, collection form and use, and (3) amount and frequency of collection of A. kerchovei. This agave is known as cacayas and rabo de león. The structures used are the flower buds ('capullos') as food for both humans and livestock of sheep and goats, the form of use is very varied and collecting is particular. Respondents indicated that their collect sites are close to their settlements and collections are done during the months of October to December. During this period seven quiotes are harvested, going on more than five occasions. Information on A. kerchovei is limited, so that this study extends their traditional knowledge and cultural importance.
\end{abstract}

Key words: agaves, cacayas, ethnobotany, rabo de león.

A lo largo del tiempo se han establecido interrelaciones entre los diversos grupos humanos con su medio, lo cual ha sido objeto de estudio con el fin de ampliar los conocimientos en torno al aprovechamiento e importancia cultural, social y económica de los recursos vegetales, tomando en cuenta sus características biológicas, ecológicas (GranadosSánchez, 1993) y etnobotánicas. El conocimiento tradicio- nal de los grupos humanos y el manejo de los recursos que les ofrecen los ecosistemas es útil en al ámbito científico, debido a que permite llevar a cabo una mejor gestión de estos recursos, especialmente para los escasos o amenazados $\mathrm{y}$, para proponer acciones a favor de la conservación de la biodiversidad (Toledo, 1988).

Actualmente, en el Valle de Tehuacán-Cuicatlán existen 
ocho diferentes grupos étnicos: Chinantecos, Cuicatlecos, Ixcatecos, Mazatecos, Mestizos, Mixtecos, Nahuas y Popolacas, quienes son poseedores de una rica herencia que ha vivido, usado y modificado el medio a través del tiempo (Dávila et al., 2002). Dentro de la Reserva de la Biosfera del Valle de Tehuacán-Cuicatlán se han registrado 1,608 especies útiles, de las cuales 610 han sido catalogadas en diversas formas de uso, las principales como: alimento (257), forraje (253), medicina (203) y ornamentales (180), entre otras. Las especies utilizadas pertenecen a 101 familias, entre las cuales resaltan con el mayor número, Cactaceae (62), Asteraceae (52), Fabaceae (42), Crassulaceae (36) y Agavaceae (23; Blancas et al., 2012).

Se han documentado algunos aprovechamientos del Aga$v e$ en el Valle de Tehuacán-Cuicatlán, tales como: elaboración de mezcal a partir del tallo de A. angustifolia Haw., A. karwinskii Zucc., A. marmorata Roezl, A. potatorum Zucc., y A. salmiana Otto ex Salm; para construcción de techos, cercos y encierros mediante el acomodo de quiotes (escapos florales) de A. kerchovei Lem., A. marmorata, A. potatorum, A. salmiana, A. scaposa Gentry y A. triangularis Jacobi; medicinal, por medio del aprovechamiento de las hojas de A. marmorata y A. scaposa como compresas; obtención de fibra de las hojas de A. kerchovei (García-Valenzuela, 2011); celebraciones religiosas con los escapos de A. marmorata (Arizaga y Ezcurra, 2002; Jiménez-Valdés et al., 2009); y alimento a partir de los botones florales de A. kerchovei (García-Valenzuela, 2011), A. marmorata, A. potatorum, A. salmiana (Blancas et al., 2010), A. scaposa y A. triangularis. Cabe señalar que la mayoría de estos aprovechamientos se realizan in situ (Blancas et al., 2010; Paredes-Flores et $a l ., 2007)$ y no tienen importancia económica.

La escasa información acerca de Agave kerchovei (Figura 1A) indica que es conocido regionalmente como cacayas, rabo de león o maguey ixtle, es un recurso alimenticio de gran importancia para sus pobladores (datos sin publicar); pero a la fecha, se carece de un estudio etnobotánico específico de la especie, por ello se considera pertinente ampliar la información del conocimiento tradicional sobre la forma de aprovechamiento de las inflorescencias de esta especie. Este estudio pretendió obtener información de las estructuras de la inflorescencia que son aprovechadas, las formas de uso, temporalidad y frecuencia de recolecta. La información resultante permitió analizar la situación actual de la especie como recurso alimentario para las localidades que pertenecen al Valle de Tehuacán-Cuicatlán.

\section{Materiales y métodos}

Área de estudio. Se eligieron dos localidades dentro de la Reserva de la Biosfera del Valle de Tehuacán-Cuicatlán, en las cuales se tomaron en cuenta los antecedentes y las visitas previas donde se localizaron poblaciones de Agave kerchovei.
San Rafael pertenece al municipio de Coxcatlán, que se encuentra ubicado en el estado de Puebla (coordenadas: $18^{\circ}$ 11 ' 42.86" N y $97^{\circ} 07^{\prime} 15.68$ "O). El clima es de semi-árido a árido, altitud de $1,217 \mathrm{~m}$ s.n.m., temperatura media anual de $22{ }^{\circ} \mathrm{C}$, presenta lluvias en verano con una precipitación total anual de 300 a 1,100 mm (INEGI, 2009). Su población cuenta con un poco más de 300 personas que habitan 63 hogares. La mayoría se dedica al cultivo de maíz (Zea mays L.), frijol (Phaseolus spp.), calabaza (Cucurbita spp.), melón (Cucumis melo L.), sandía (Citrullus spp.) y caña de azúcar (Saccharum officinarum L.), éste último es el más importante en la localidad; además recolectan otros productos en su entorno natural (Rosas-López, 2003; Blanckaert et al., 2004). Dentro de su actividad pecuaria resalta el fomento y la cría de ganado caprino y ovino, el cual pace en los montes circundantes. Por otra parte, la mayoría de las mujeres se dedican a las actividades del hogar (Blanckaert et al., 2004).

San Gabriel Casa Blanca (en adelante San Gabriel) es la Agencia Municipal perteneciente al Municipio de San Antonio Nanahuatipam en el estado de Oaxaca. Se ubica a $18^{\circ}$ $09^{\prime} 14.6^{\prime \prime} \mathrm{N}$ y $97^{\circ} 08^{\prime} 38^{\prime \prime} \mathrm{O}$ a una altitud de $946 \mathrm{~m}$ s.n.m. El clima es transicional semi-árido a árido, la temperatura promedio anual varía entre 25 y $30{ }^{\circ} \mathrm{C}$, lluvias en verano, precipitación total anual de 300 a $800 \mathrm{~mm}$. (INEGI, 2008). Su población es de 372 habitantes (INEGI, 2005) en un poco más de 100 hogares. La actividad agrícola principal es el cultivo de caña de azúcar, seguido del melón y maíz. También en esta localidad se identifica la actividad pecuaria de ovejas y cabras, principalmente de traspatio. Otras actividades que comprenden a la minoría de la población son el comercio, albañilería y servicio de transporte. Las mujeres particularmente realizan las actividades hogareñas (Moreno-Bautista, 2008).

Estudio etnobotánico. Para obtener información acerca del aprovechamiento de Agave kerchovei, los entrevistados fueron hombres y mujeres mayores a 20 años (Cuadro 1), considerando que la población adulta está entre los 24 y 58 años (Blanckaert, 2001) y cuya actividad para las mujeres es ser amas de casa encargadas de cocinar y, para los hombres, ser campesinos activos en las labores y rutinas del campo, quienes se ocupan de la recolección. Se usó un cuestionario estructurado de once preguntas (Cuadro 2), diseñado para obtener información en torno al aprovechamiento del esca-

Cuadro 1. Número de hombres y mujeres entrevistados por categorías de edad.

\begin{tabular}{ccccc}
\hline LOCALIDAD & HOMBRES & EDAD & MUJERES & EDAD \\
\hline San Rafael, Puebla & 10 & $26-70$ & 21 & $21-75$ \\
$\begin{array}{c}\text { San Gabriel Casa } \\
\text { Blanca, Oaxaca }\end{array}$ & 12 & $38-82$ & 25 & $25-70$ \\
\hline
\end{tabular}


po y los botones florales (capullos) del agave. El cuestionario inicia con la identificación de la especie de Agave kerchovei y nombres con los que se conoce, mediante fotografías previamente tomadas en San Rafael, Puebla; la segunda sección está enfocada a la forma de aprovechamiento y la tercera, a la ubicación de los sitios de recolecta, intensidad de aprovechamiento, temporalidad y destino de los productos cosechados. Los datos consignados se organizaron para ambas localidades (Cuadro 2), incluyendo el total de entrevistados, la frecuencia de respuesta por pregunta $(\mathrm{F})$ y, por medio del método de consenso de los informantes, se calculó el nivel de fidelidad (NF), el cual permitió conocer la importancia del recurso según la respuesta individual de los entrevistados (Friedman $e t$ al., 1986; Avendaño et al., 2006):

$$
\mathrm{NF}(\%)=I_{p} / I_{u}(100)
$$

Donde: $I_{p}$ es el número de informantes que respondieron cada pregunta, $I_{u}$ es el número total de informantes en cada localidad.

\section{Resultados}

Etnobotánica de Agave kerchovei. Se desconoce el origen de la denominación tradicional de A. kerchovei como cacayas. Por su parte, el nombre de rabo de león es por la semejanza en la forma entre la inflorescencia y la cola de dicho mamífero. Durante las entrevistas quedó claro que los botones son las cacayas y la planta es el rabo de león; aunque hay quienes usan el nombre de cacayas también para toda la planta.

Las entrevistas realizadas confirmaron que existen pocas poblaciones silvestres de Agave kerchovei. Las personas entrevistadas de San Rafael, Puebla, ubicaron como sus sitios de recolecta "Las Compuertas" y "El Río", resaltando que las poblaciones conocidas por ellos son pocas, pequeñas, lejanas y de difícil acceso. Por otra parte, en San Gabriel, Oaxaca, se recolecta rabo de león en "Tecolotiopa", "La Cruz de Mica", "El Río" y "Calapa".

En San Rafael el 65\% de los entrevistados conoce a este agave solamente como cacayas y $16 \%$ como rabo de león, el resto (19\%) lo conoce con los dos nombres; mientras que en San Gabriel el 32\% de los entrevistados lo conoce sólo como rabo de león y el $22 \%$ como cacayas, el $46 \%$ con ambos, indicando que es indistinto. Los órganos y las estructuras más utilizadas son los botones y el escapo. En San Rafael, la mayoría (77\%) utiliza botones, $3 \%$ quiote y $16 \%$ ambos. En San Gabriel, el 51\% utilizan los botones y $49 \%$ ambos (Cuadro 2).

El aprovechamiento de los botones es alimenticio (Figura 1B), $40 \%$ de los pobladores de San Gabriel lo indicaron como el único uso, el $60 \%$ además lo emplean como forraje para el ganado caprino u ovino (Figura 1C). Por otra parte, el quiote lo usan para la elaboración de mangos de escobas,

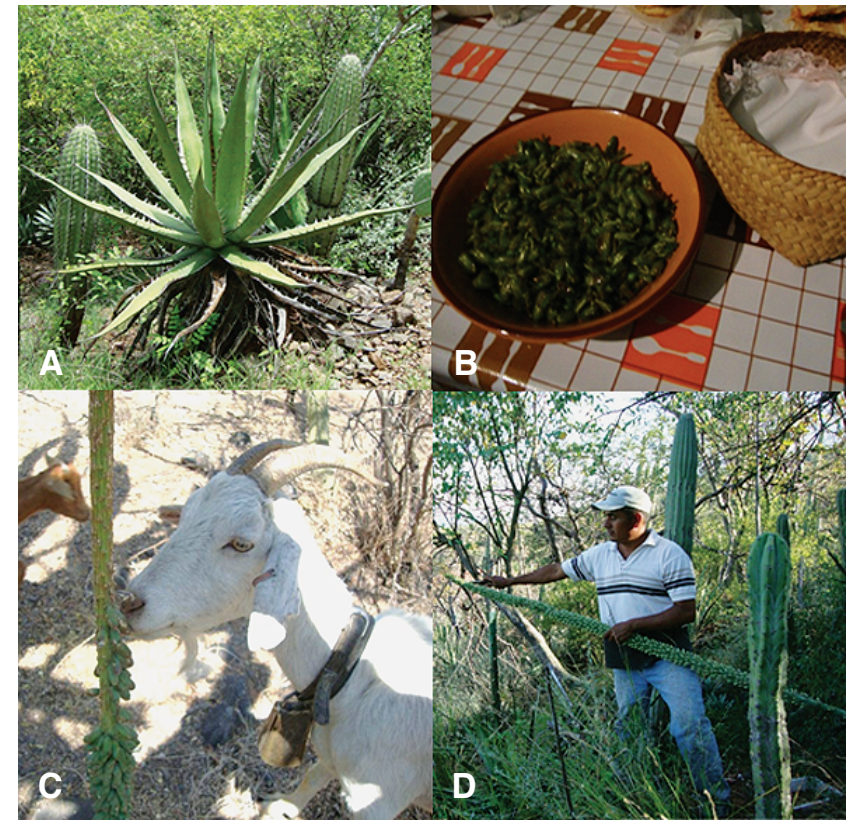

Figura 1. A. Planta de Agave kerchovei; B. Cacayas cocinadas; C. Cabra alimentándose de cacayas; D. Poblador cortando una porción del ápice del quiote para posteriormente separar las cacayas en su hogar.

también construyen cercos y corrales, y la roseta es utilizada como ornamento.

Los informantes de San Rafael utilizan los botones para alimento principalmente (94\%), y como dato adicional, solo el 3\% mencionó el uso de las hojas como productoras de fibra, materia útil para elaborar cordeles, canastos, ayates y estropajos, aunque refieren que dicho aprovechamiento está en desuso.

La forma de consumir los botones es variada y depende del gusto de las personas. En general, los botones tiernos se preparan asados, fritos, hervidos y en conserva (Figura 1B), 84 y 95\% en San Rafael y San Gabriel, respectivamente; a la vez, sólo el 13 y $5 \%$ en cada comunidad utilizan el quiote para la elaboración de escobas y construir cercos y corrales. El quiote conforme se deshidrata se vuelve ligero, pero a la vez, muy resistente por la cantidad de fibras que contiene. La totalidad de los entrevistados en ambas comunidades indican que la recolecta del quiote y los botones se obtienen únicamente para autoconsumo, no se comercializa.

$\mathrm{Al}$ recolectar el quiote existen dos variantes: en la primera, si requieren los botones florales sólo recolectan la porción del escapo que los contiene (Figura 1D); en la segunda, se obtiene la totalidad del quiote porque además de los botones, al quiote le dan los usos ya referidos. El 100\% de las personas entrevistadas de San Rafael obtiene y transporta el quiote completo. En San Gabriel sólo el 89\%, el 11\% restante dobla o corta el quiote después de tomar los botones necesarios. El uso como forraje para ovicaprinos es in situ. 
Cuadro 2. Respuestas obtenidas durante la aplicación de las entrevistas para recabar información general en torno a la importancia y aprovechamiento de Agave kerchovei en las localidades bajo estudio. F = Frecuencia, NF = nivel de fidelidad.

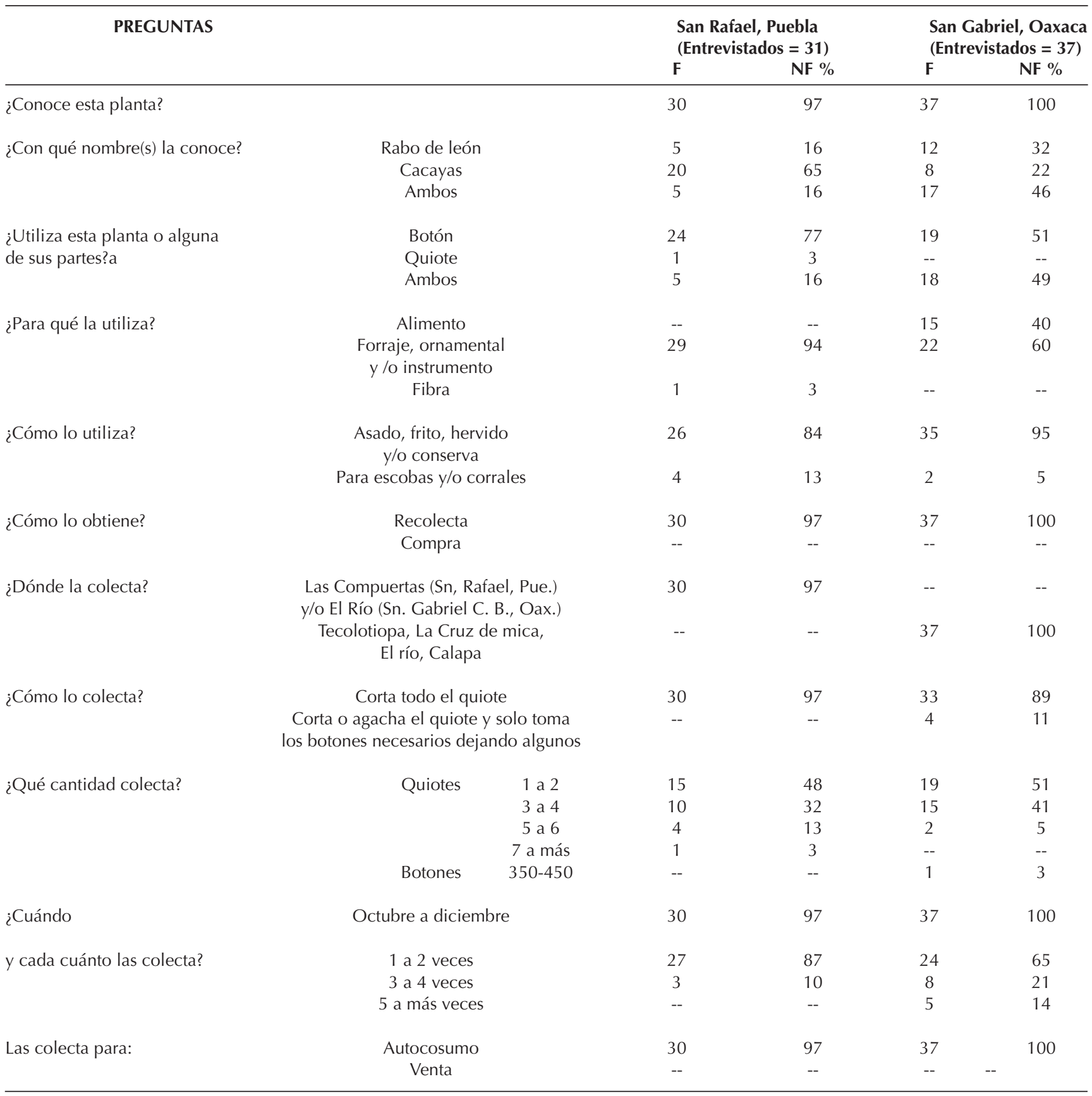

Se prefiere comer los botones y no las flores (en antesis y maduros para el proceso reproductivo), para lograr un sabor agradable (no amargo). Además, consideran que el buen sabor de las cacayas dependerá de la persona quien las recolecte; para esto, la mayoría sigue un procedimiento, el cual consiste en cortar una porción apical de la inflorescencia, unos $15 \mathrm{~cm}$. (Figura 1D). También les parece importante la persona (generalmente ama de casa) que cocinará y la forma en que las procesará, para lo cual cuentan con una amplia variedad de opciones gastronómicas características de su cultura, éstas van desde sólo hervir las cacayas, freírlas en aceite con limón y sal, combinarlas con mole, con huevo, en caldo y otros modos culinarios.

El periodo de recolecta de quiotes y botones, en ambas localidades, ocurre en el otoño, durante este periodo de floración varía la frecuencia y cantidad de recolecta, aunque se estima que realmente la demanda es considerable. Los pobladores de San Rafael revelan que durante la estación, 
el $87 \%$ recolecta cacayas máximo dos veces y el $10 \%$ hasta cuatro veces. Por el contrario, en San Gabriel el 65\% recolecta una o dos veces, $21 \%$ hasta cuatro y el $14 \%$ más de cinco veces. La frecuencia de recolecta es acorde con el gusto de la gente por las cacayas.

\section{Discusión}

La información etnobotánica derivada del presente estudio ratifica la importancia cultural de Agave kerchovei dentro del Valle de Tehuacán, con base en el conocimiento individual de los pobladores en torno a esta planta (Reyes-García et al., 2007). La importancia de un recurso se refleja en el número de personas que lo usufructúan y las formas de aprovechamiento, como lo consignan Avendaño et al. (2006) para el caso del fruto de Ceiba aesculifolia subsp. parvifolia (Rose) P.E. Gibbs \& Semir en dos municipios dentro del Valle de Tehuacán, y como lo documentara Estomba et al. (2006) acerca del conocimiento tradicional de plantas medicinales silvestres y su aplicación en diversos padecimientos en una comunidad Mapuche.

Agave kerchovei destaca por el consumo de los botones florales, al igual que otras especies de Agave de diversas regiones del país. En el Valle de Tehuacán se documenta este mismo aprovechamiento para A. marmorata, A. salmiana y A. scaposa (Blancas et al., 2010); específicamente en comunidades del municipio de Coxcatlán las especies A. kerchovei (cacayas) y A. potatorum (cacayas o tibilos, García-Mendoza, 2010). Agave potatorum también es aprovechado para la elaboración de mezcal, proceso que implica la interrupción del desarrollo floral (Pérez-Negrón y Casas, 2007). Por otra parte Arias et al. (2000) mencionan el uso alimentario de A. macroacantha (cacayas de segunda) y A. stricta Salm-Dyck (cacayitas), en la zona de Zapotitlán de las Salinas, Puebla. En el estado de Hidalgo se refieren especies silvestres tales como: A. albicans Jacobi, A. lechuguilla Torrey, A. striata Zucc. y dos especies cultivadas, A. mapisaga Trel. y A. salmiana, de las cuales, además del consumo y gran valor económico por su venta en las plazas de la región, se obtiene aguamiel para la elaboración de pulque (CONANP, 2003).

El vocablo cacaya se aplica a diferentes especies. En el caso de A. kerchovei los entrevistados mencionaron cacayas, en San Rafael, Puebla y rabo de león en San Gabriel Casa Blanca, Oaxaca. Esta situación corresponde con lo mencionado por Gentry (1982), quien advierte que en Mitla y Huajuapan es llamado rabo de león y en la región de Tehuacán como cacaya o maguey pochonal.

La recolección de cacayas es un evento esperado cada año, los pobladores afirman que realizan esta práctica durante el periodo de octubre a diciembre, mientras que en la literatura se documenta que la floración de Agave kerchovei ocurre de octubre a enero (Gentry, 1982; Arias-Toledo et al., 2000). Este recurso no reditúa en un ingreso económico por no ser comercializado, ya que es manejado únicamente in situ, como el 23\% de las especies vegetales útiles en el Valle de Tehuacán (Blancas et al., 2010); es por esto que acuden a los sitios más cercanos a sus hogares o como un acto de oportunidad, recolectan las cacayas durante el desarrollo de otra actividad en el campo. El aprovechamiento integral del pedúnculo floral concluye con la fabricación de mangos de escobas, cercos perimetrales, material de construcción, etc., usos con valor social aplicado a la vivienda (GarcíaValenzuela, 2011).

Es importante resaltar que el aprovechamiento de las estructuras florales debe tomarse en cuenta en evaluaciones de impacto sobre las poblaciones de Agave kerchovei, ya que de los botones florales y futuras flores en antesis depende la reproducción sexual, evento que sólo ocurre una vez en la vida de los agaves, por ser plantas semélparas (Gentry, 1982; Nobel, 1988). Por lo tanto, la forma de aprovechamiento para consumo de las estructuras reproductivas podría tener efectos negativos para las poblaciones de A. kerchovei, ya que se interviene en el flujo genético (Barrett, 2002) y, en consecuencia, se afecta la permanencia de las poblaciones y el futuro de la especie. Se documenta que A. kerchovei, tiene mayor recurrencia de multiplicación asexual que de reproducción sexual, pues en ésta influyen una serie de factores bióticos, así como el manejo dentro de las comunidades (Brena-Bustamante, 2012).

Consideramos que estudios demográficos revelarían si las poblaciones pueden cubrir esa demanda alimentaria y de aprovechamiento múltiple, permitiendo que la dinámica poblacional de la especie continúe sin afectarse en términos de supervivencia. Estamos de acuerdo con Prance (1995) y Davidson-Hunt (2000), quienes proponen como una necesidad incorporar principios ecológicos en el desarrollo de las investigaciones etnobotánicas tradicionales que aboguen por la integración de diversos factores, procesos y experiencias que permitan ampliar, establecer y planear el manejo y conservación de los recursos y sus servicios a nivel local y regional (Pérez-Negrón y Casas, 2007), inclusive en las pertenecientes a Áreas Naturales Protegidas, como es el caso de la situación geográfica en la que concurre Agave kerchovei.

\section{Agradecimientos}

Al Consejo Nacional de Ciencia y Tecnología (CONACyT) por la beca otorgada (235672) para la realización de este estudio. Al Colegio de Postgraduados que me dio la oportunidad de desarrollarme académicamente y fue el lugar donde se dirigió la presente investigación, y al proyecto "Conservación de semillas de plantas útiles de San Rafael, Municipio de Coxcatlán, Puebla, MGU-Useful Plants Project México", el cual se desarrolló de 2007 a 2011 con el apoyo de los Jardines Botánicos Reales de Kew y bajo la dirección de Rafael Lira Saade en la Facultad de Estudios 
Superiores Iztacala, UNAM, por facilitar los medios para llevar a cabo este estudio. Se reconoce ampliamente a los revisores de este manuscrito por sus acertados y enriquecedores comentarios.

\section{Literatura citada}

Arias T.A.A., Valverde V.M.T. y Reyes S.J. 2000. Las Plantas de la Región de Zapotitlán Salinas, Puebla. Instituto Nacional de Ecología. Universidad Nacional Autónoma de México, México, D.F.

Arizaga S. y Ezcurra E. 2002. Propagation mechanisms in Agave macroacantha (Agavaceae), a tropical arid-land succulent rosette. American Journal of Botany 89:632-641.

Avendaño A., Casas A., Dávila P. y Lira R. 2006. Use forms, management and commercialization of "pochote" Ceiba aesculifolia (H. B. \& K.) Britten \& Baker f. subsp. parvifolia (Rose) P. E. Gibbs \& Semir (Bombacaceae) in the Tehuacán Valley, Central Mexico. Journal of Arid Environments 67:15-35.

Barrett S.C.H. 2002. Evolution of sex: The evolution plant sexual diversity. Nature Reviews Genetics 3:274-284.

Blancas J., Casas A., Rangel-Landa S., Moreno-Calles A., Torres I., Pérez-Negrón E., Solís L., Delgado-Lemus A., Parra F., Arellanes Y., Caballero J., Cortés L., Lira R. y Dávila P. 2010. Plan management in the Tehuacán-Cuicatlán Valley, México. Economic Botany 64:287-302.

Blanckaert I. 2001. An ethnobotanic survey of homegardens in San Rafael Coxcatlán, Valley of Tehuacán-Cuicatlán, Mexico. Master of Science in Tropical Agriculture. Faculty of Agricultural and Applied Biological Sciences. Catholic University of Leuven. Leuven. 83 pp.

Blanckaert I., Swennen R.L., Paredes-Flores M., Rosas-López R. y Lira-Saade R. 2004. Floristic composition, plant uses and management practices in homegardens of San Rafael Coxcatlán, Valley of Tehuacán-Cuicatlán, Mexico. Journal of Arid Environments 57:179-202.

Brena-Bustamante P. 2012. El aprovechamiento y la estructura poblacional de Agave kerchovei Lem. en Tehuacán-Cuicatlán, México. Tesis de Maestría. Colegio de Postgraduados, Campus Montecillo, Texcoco, Estado de México. 84 pp.

CONANP (Comisión Nacional de Áreas Naturales Protegidas). 2003. Programa de manejo de la reserva de la Biosfera Barranca de Metztitlán, México. Dirección General de Manejo para la Conservación. México, D.F.

Dávila P., Arizmendi M. C., Valiente-Banuet A., Villaseñor J.L., Casas A. y Lira R. 2002. Biological diversity in the Tehuacán-Cuicatlán Valley, México. Biodiversity and Conservation 11:421-442.

Davidson-Hunt I. 2000. Ecological ethnobotany: stumbling toward new practices and paradigms. MASA Journal 16:1-13.

Estomba D., Ladio A. y Lozada M. 2006. Medicinal wild plant knowledge and gathering patterns in a Mapuche community from North-western Patagonia. Journal of Ethnopharmacology 103:109-119.

Friedman J., Yaniv Z., Dafni A. y Palewitch D. 1986. A prelimi- nary classification of the healing potential of medicinal plants, based on a rational analysis of an ethnopharmacological field survey among Bedouins in the Negev Desert, Israel. Journal of Ethnopharmacology 16:275-287.

García-Mendoza A. 2010. Revisión taxonómica del complejo Agave potatorum Zucc. (Agavaceae): nuevos taxa y neotipificación. Acta Botanica Mexicana 91:71-93.

García-Valenzuela M.A. 2011. Etnoecología de los agaves (Agavaceae) en la comunidad Ngiwa (Popoloca) de los Reyes Metzontla, Puebla, México. Tesis de Maestría. Colegio de Postgraduados, Campus Montecillo. Texcoco, Estado de México. 122 pp.

Gentry H. S., 1982. Agaves of Continental North America. University of Arizona Press, Tucson.

Granados-Sánchez D.1993. Los Agaves en México. Universidad Autónoma de Chapingo, Texcoco.

INEGI, 2005. Marco Geoestadístico Municipal. Versión 3.1. $<\mathrm{http}: / /$ mapserver.inegi.org.mx/data/mgm/> (consultado $5 \mathrm{de}$ abril de 2012).

INEGI, 2008. Prontuario de Información Geográfica municipal de los Estados Unidos Mexicanos. San Antonio Nanahuatipam, Oaxaca. Clave geoestadística 20109. <http://www3.inegi.org. $\mathrm{mx} /$ sistemas/mexicocifras/datos-geograficos/20/20109.pdf> (consultado 22 de febrero de 2012).

INEGI, 2009. Prontuario de Información Geográfica municipal de los Estados Unidos Mexicanos. Coxcatlán, Puebla. Clave geoestadística 21035. <http://www.inegi.org.mx/sistemas/ mexicocifras/datos-geograficos/21/21035.pdf> (consultada 4 de julio de 2012).

Jiménez-Valdés M., Gódinez-Álvarez H., Caballero J. y Lira R. 2009. Population dynamics of Agave marmorata Roezl. under two contrasting management systems in Central Mexico. Economic Botany 64:149-160.

Moreno B.J.L. 2008. Diagnóstico municipal 2008. Trienio 20082010. San Antonio Nanahuatipam, Teotitlán de Flores Magón, Oaxaca.

Nobel P.S.1988. Environmental Biology of Agaves and Cacti. Cambridge University Press, Nueva York.

Paredes-Flores M., Lira S.R. y Dávila A.P.D. 2007. Estudio etnobotánico de Zapotitlán Salinas, Puebla. Acta Botanica Mexicana 79:13-61.

Pérez-Negrón E. y Casas A. 2007. Use, extraction rates and spatial availability of plant resources in the Tehuacán-Cuicatlán Valley, Mexico: The case of Santiago Quiotepec, Oaxaca. Journal of Arid Environments 70:356-379.

Prance G. 1995. Ethnobotany today and in the future. En: Schultes R.E. y von Reis S. Eds. Ethnobotany: Evolution of a Discipline, pp. 61-68, Timber Press, Portland.

Reyes-García V., Martí N., McDade T., Tanner S. y Vadez V. 2007. Concepts and methods in studies measuring individual ethnobotanical knowledge. Journal of Ethnobiology 27:182-203.

Rosas-López R. 2003. Estudio etnobotánico de San Rafael-Coxcatlán, Tesis de licenciatura. Facultad de Estudios Superiores Iztacala, Universidad Nacional Autónoma de México, México. 95 pp.

Toledo V.M. 1988. La diversidad biológica de México. Ciencia y Desarrollo 81:17-50.

Recibido: 18 de marzo de 2012

Aceptado: 6 de septiembre de 2012 